\title{
Categorization of Silkworm Based on Chitin Glands using Image Processing
}

\author{
Shreyas S., Simhadri Govindappa, C. G. Raghavendra, Vinayak Shastri, Yathin Patil
}

\begin{abstract}
This paper demonstrates a prototype for highly accurate identification of the silkworm pupa (Bombyx mori) gender using optical property. The methodology is to optical beam in the near infrared spectrum that can effectively and safely penetrate the body of a silkworm pupa. After the illumination, some of the basic operations of image processing like image thresholding, contour detection, blob filtering and image inversion processes are applied to remove the unwanted image noises and at the same time highlighted the gland that distinguishes the gender in silkworm. The proof of concept is experimentally done using three $633 \mathrm{~nm}$ wavelength Light emitting diodes (LED's), a pi camera, and a computer. Some of the key features of this method include ease of implementation with cost reduction and high accuracy.
\end{abstract} Pupa.

Keywords: Chitin Gland, Gaussian Blurring, Thresholding,

\section{INTRODUCTION}

The unintentional discovery of silk by the Chinese empire almost 5000 years ago marked the beginning of the most prominent industries in mankind, the sericulture industry. Even today sericulture plays an important role in the economic growth of developing nations. As evident in the 2017-18 report published by the Central Silk Board, Ministry of Textiles which describes the sericulture's contribution to the economy and lists silkworm cocoon production as one of the most important agricultural commodities in India with 1600 million metric tons production (CSB, 2018) per year.

High quality raw silk is obtained by cross breeding of silkworms, sex identification processes [1] are used to identify male and female silkworms. The strong and healthy breed of silkworms can be separated with the assistance of gender identification and classification processes. In the past four decades, a number of silkworm pupa gender separation techniques have been proposed and demonstrated with varying success rates. Most accurate one among them is Deoxyribonucleic Acid (DNA) analysis.

Revised Manuscript Received on December 15, 2019.

Shreyas S., Department of Electronics and Communication Engineering, Ramaiah Institute of Technology, Bangalore, India.

Simhadri, Department of Electronics and Communication Engineering, Ramaiah Institute of Technology, Bangalore, India.

C. G. Raghavendra, Assosciate Professor, Department of E\&C, Ramaiah Institute of Technology, Bangalore, India.

Vinayak Shastri, Department of Electronics and Communication Engineering, Ramaiah Institute of Technology, Bangalore, India.

Yathin V. Patil, Department of Electronics and Communication Engineering, Ramaiah Institute of Technology, Bangalore, India.
Other destructive methods such as scanning with high frequency radio waves are not used practically as their set up time and cost are more than the affordable limit of farmers in most the developing countries and are destructive in nature. The commonly used method, is the visual inspection of silkworm pupa. The low cost and non-destructive nature of this method has a wide spread adoption in the rearing community to determine the gender of silk pupa. Clearly the drawback of this method is that it requires a highly skilled and well trained workers, thus making it more labor intensive.

The DNA and Ribonucleic Acid(RNA) based gender classification of silkworm is done based on certain chemical reactions on silkworms [2]. Magnetic and near infrared (NIR) spectroscopy are two promisingly non-destructive approaches for determining the gender of silk pupa. These two methods are not adaptable as the speed is less and the system costs are high. Optical fluorescent imaging is another interesting method.

Female and male silkworm pupa can be separated under the illumination of ultra-violent light sources [3]. However, only limited species can be separated by this method [4] as there are improvements in the silkworm cocoon through dye-modified diet and cross breeding of silkworm. As a solution for the above encountered problems, in this paper it is proposed and experimentally demonstrated how to detect the gender of the silkworm using optical penetration method with a very high accuracy.

In the proposed method we are using four basic image processing steps for analysis process covering thresholding of image, gray scale conversion, morphological transforms and blob filtering, because of which a slow identification time is obtained. This also ensures the low response time. It is evident from the above discussion that, to have a faster response time it is desirable to reduce the number of image processing operations needed during the identification of silk pupa gender. In this paper, it is experimentally demonstrated that by simple Normalized Cross Correlation (NCC) based pattern matching approach the gender of the silk pupa can be realized in a faster and efficient manner. Some of the other key features of optical penetration based silkworm gender identification include high accuracy, ease of implementation, adaptive learning ability, and low component counts and cost

\section{METHODOLOGY}

The silk pupa was placed under $600 \mathrm{~nm}$ wavelength light red light as shown in Fig. 1 and the images of the silk pupa were taken. 
These images were further subjected to an image processing algorithm to extract out the pupa contour from the noisy background through thresholding and morphological transforms. The setup diagram for the proposed method to identify the gender of silkworm pupa by optical penetration is as shown in Fig. 1.where silkworm pupa is placed on a slab under a 2D pi camera, the slab is designed in such way that there is a slot to place a series of LED's or other relevant light sources that radiate red light. The silk pupa is exposed to the radiation of high frequency in the visible range(red) that are being emitted by series of the LED's or light source placed in the provided slots of the slab. These high frequency rays penetrate through shell and exoskeleton of the silkworm. Meanwhile, some of the transmitted light is blocked by an organ called the chitin gland, this creates corresponding dark regions in the image of the female silkworm pupa as the organ is only found in the female silkworms and not in male silkworms. The electronic system unit with powered batteries is used to regulate the series of light sources and camera. The image is also analyzed in the same functional unit. As there is variation in size and skin thickness of both female and male silkworm pupa the light intensity of sources is gradually increased until the chitin gland is clearly observed in the shadow region associated with it. The intensity of light sources reaches the maximum level if there is no such shadow region.

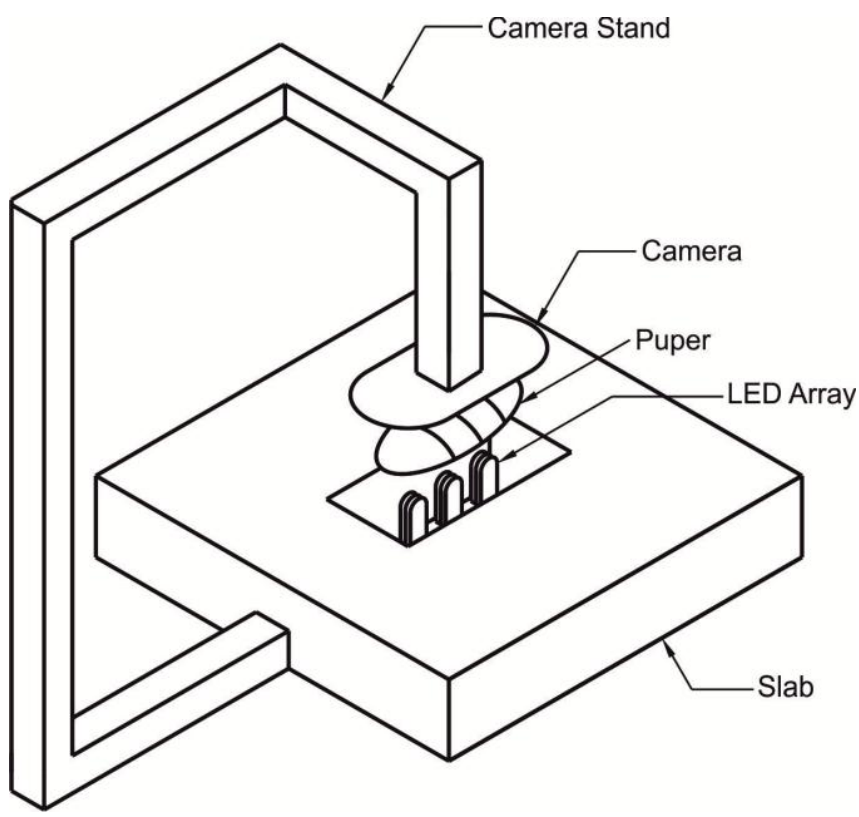

Fig. 1.Schematic of the optical imaging device

\section{DATA COLLECTED}

Using the setup described above, a good number of pupa images were collected and were annotated or classified as male or female by experts.

These samples of data were then used to build an image processing algorithm to correctly classify the male and female pupas with high accuracy. Some of the collected sample images as shown in Fig. 2 and Fig. 3.
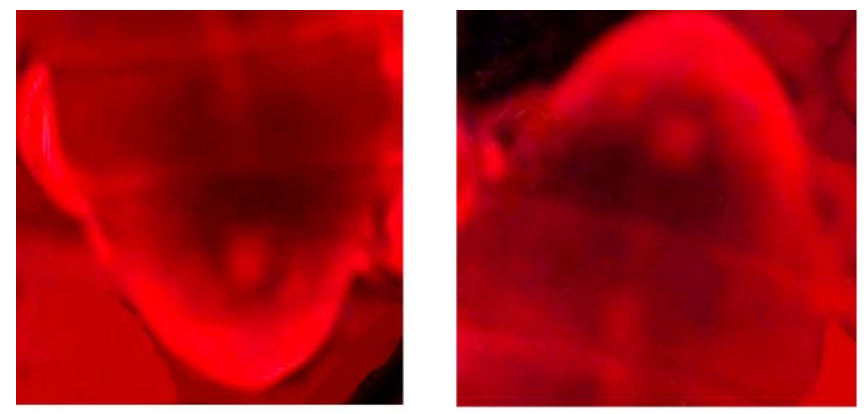

Fig. 2.Sample Images of Female Pupa
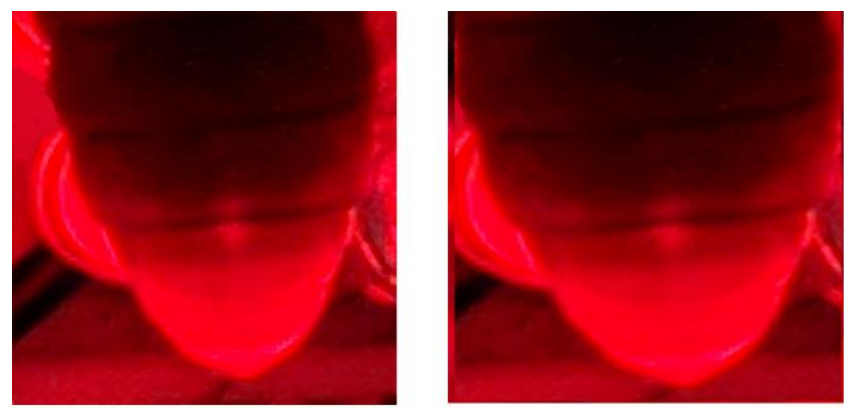

Fig. 3.Sample Images of Male Pupa

\section{ALGORITHM}

The image obtained from the setup shown is Fig.1, is subjected to image processing techniques to remove noise, perform thresholding followed by blob detection to identify the chitin gland in the image. Based on this the silk pupa are classified as male or female.

Fig. 4 shows the flowchart of the algorithm. The image is first Gaussian blurred, resized, converted to gray scale and then binarized by thresholding. This extracts the pupa from the background noise. Then the region of interest is extracted and morphological operations are performed to further remove the noise from the image. Finally hull detection followed by blob detection using contours is used to detect the blobs. These contours are then filtered and sorted by area. These are used to determine the existence of a chitin gland to classify as male or female.

$$
f_{c}(x, y)=1 \text { if }(x, y)=0 \text {, and } f c(x, y)=0 \text { if } f(x, y)-1
$$

The intersection $h=f \cap g$ of two binary images $\mathrm{f}$ and $\mathrm{g}$ :

$h(x, y)=1$ if $f(x, y)=1$ and $g(x, y)=1$

and $h(x, y)=0$ otherwise

$$
h(x, y)=1 \text { if } f(x, y)=1 \text { or } g(x, y)=1
$$

and $h(x, y)=0$ otherwise 


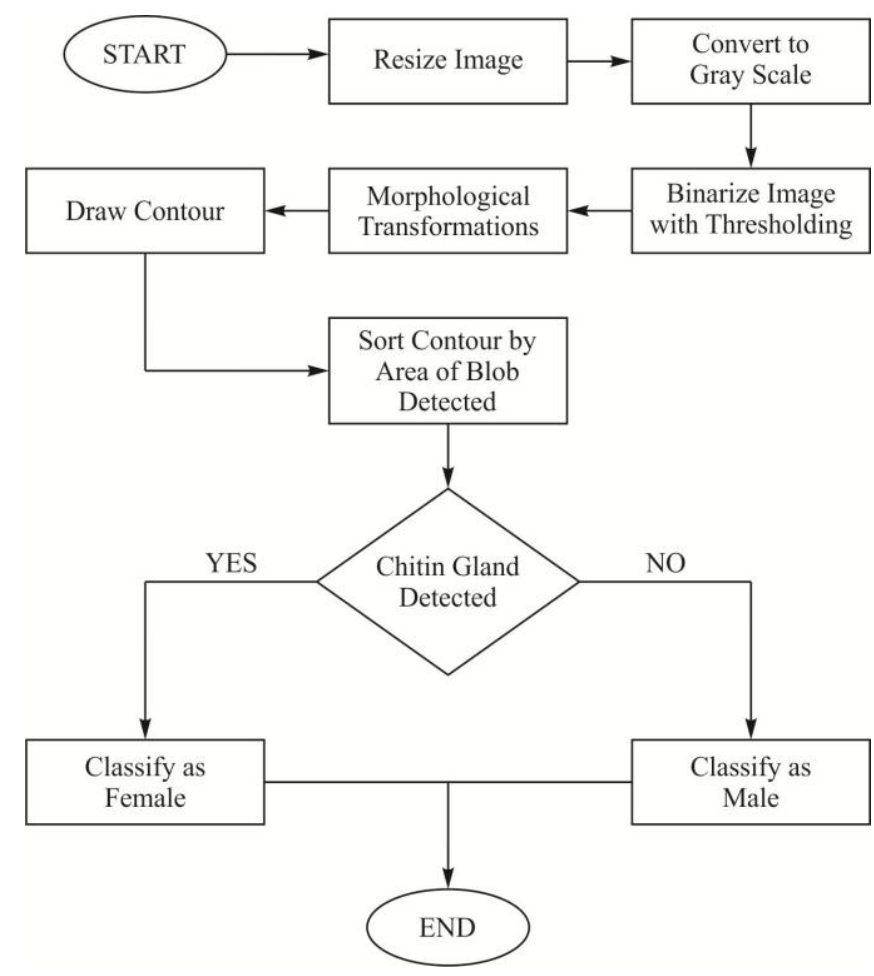

Fig. 4.Flow Chart of the Implemented Algorithm

\section{RESULT AND DISCUSSION}

After the images were taken from the setup as shown in Fig. 1 the image processing algorithms were applied to extract the features required. After obtaining the required features, we will compare and draw conclusion from this, suggesting whether the pupa is male or female.

In this section we are computing the exact accuracy of the method implemented and the corresponding statistical comparison.

\section{A. Determination of Gender}

The below table shown compares the accuracy of classifying the silkworms based on the penetration method used.

Table- I:

\begin{tabular}{|c|c|c|}
\hline Particulars of Pupa & Male & Female \\
\hline Total Samples & 250 & 250 \\
\hline $\begin{array}{l}\text { Correctly } \quad \text { Detected } \\
\text { Samples }\end{array}$ & 247 & 240 \\
\hline Accuracy & $98.8 \%$ & $96 \%$ \\
\hline
\end{tabular}

To extract data and identify the silkworm as male or female the proposed method follows the following general steps for both male and female:

- The images of the silkworm pupa under light of wavelength $(600 \mathrm{~nm})$ were collected from field visit were first resized to bring all the images to a uniform

- The images were then subjected to Gaussian blurring to smoothen the images. Gaussian blur was then followed by gray scaling and thresholding to get a binary image to remove the background and noise.

- After thresholding we detected blobs in the image and filter out blob corresponding to chitin gland using resolution of $(1000 * 700)$ pixels.

morphological transform and size of the blob using contour detection.

- Based on the existence of blob, corresponding chitin gland the pupa was sorted as male and female.

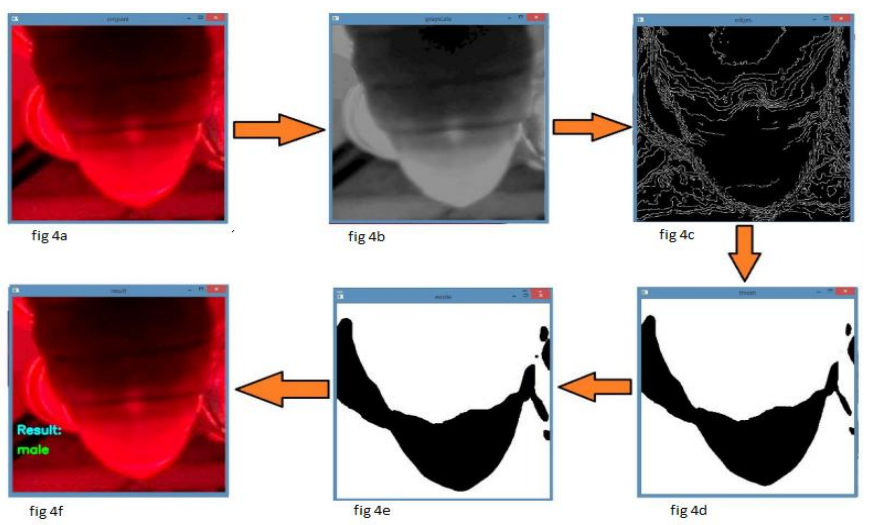

Fig. 5.Detection of Male Pupa

The image shown in Fig.5 describes how the discussed image processing methods are applied to the male pupa image.

The image shown in Fig.6 describes how the discussed image processing methods are applied to the female pupa image.

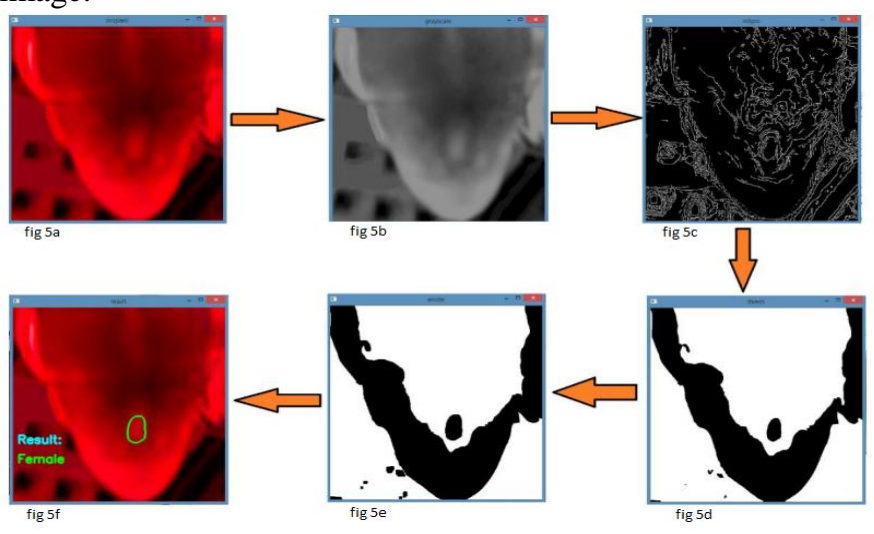

Fig. 6.Detection of Female Pupa

\section{CONCLUSION}

This paper studies how the simple pattern matching operation is used for identification of silkworm gender by optical penetration method. The proof for the concept is experimentally discussed and demonstrated by using 6 red LEDs, PI cam and a laptop as a setup. In the experiment 250 female and 250 male silkworm pupa were used in this study. A very good number of images taken out of the PI cam are randomly selected and are worked upon with various image processing techniques to extract the features like its shape and the presence of chitin glands. After this feature extraction the original image and the processed image are displayed on the Graphical User Interface (GUI).Using the system suggested, the gender identification and classification of the silkworms can obtain in less than $15 \mathrm{~ms}$ on the real time data with the best measured accuracy of $98.8 \%$ and $86 \%$ for male and female respectively, 
this indicates a high potential for deployment of this concept in the sericulture industry.

\section{ACKNOWLEDGMENT}

The authors are grateful to MSRIT Alumni Association, Bangalore for funding the project.

\section{REFERENCES}

[1] Katsuma, S, M., Fujimoto, Kiuchi, T, T., Sahara, K, \& Kawamoto. (2018). "Unique sex determination system in the silkworm, Bombyx mori: current status and beyond", Proceedings of the Japan Academy, Series B, 94(5), 205-216.

[2] Liu, L., Jin, T.,Tang, X., Chen, H., (1995). "Differentiation of male, female and dead silkworms while in the cocoon by near infrared spectroscopy". Journal of Near Infrared Spectroscopy 3, 89-95.

[3] Yu Qing, Z., Wei De, S., Xiao Hua, Y. Li Xia, Z, Yong Lei, M., Shu Qian, Y., Nai Xi, X., (2010)." Mechanism of fluorescent cocoon sex identification for silkworms Bombyx mori”. Science China Life Sciences 53, 1330-1339.

[4] Zhang, Y W., Yu, X., Shen, Yi, S., Zhou, L., Xu, N., \& Ma, Y. (2010). "Mechanism of fluorescent cocoon sex identification for silkworms Bombyx mori”. Science China Life Sciences, 53(11), 1330-1339.

[5] R. G. Srinidhi, R. N. Nikitha, Amar T., R. Harshith, and C. G. Raghavendra, "Reckoning the Hatch Rate of Multivoltine Silkworm Eggs by Differentiating Yellow Grains from White Shells Using Blob Analysis Technique", Springer 2018. Recent Findings in Intelligent Computing Techniques, Advances in Intelligent Systems and Computing 709.

[6] K. A Ray and Acharya T., Image Processing Principles And Applications, Wiley, (2005).

[7] Gonzalez, R.C. and Woods, R.E.: Digital Image Processing, 3rd edition., (2007)

\section{AUTHORS PROFILE}

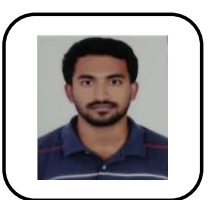

Shreyas S. holds his BE degree in Electronics and Communication Engineering from Ramaiah Institute of Technology, Bangalore. His fields of interests includes machine learning and image processing.

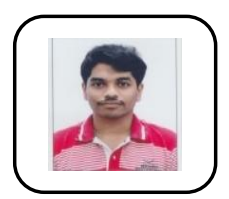

Simhadri holds his BE degree in Electronics and Communication Engineering from Ramaiah Institute of Technology, Bangalore. His fields of interests includes Deeplearing, Cryptography, Signal proceesing and Computer vision .

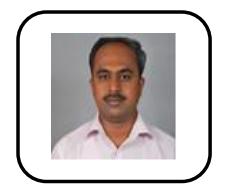

C. G. Raghavendra obtained his B.E., M.Tech and Ph.D in E\&C in 2001, 2004 and 2018 respectively. He worked as Engineer in Aeronautical Development Agency (ADA, Ministry of Defence, Govt of India) during 2001-02. Presently Working as Assosciate Professor in Ramaiah Institute of Technology, Dept of E\&C, Bangalore, India from 2004 till date. He has 14+ years of teaching experience. Completed several funded projects from VGST, Govt of Karnataka and KSCST, Bangalore. Filed two Indian patents in the area of signal processing. He has published papers in international journal.He is a fellow of IETE, Senior Member of IEEE, Member in IEI, IMAPS and ISTE.

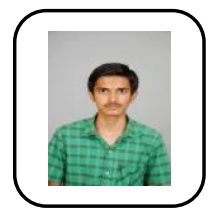

Vinayak Shastri holds his BE degree in Electronics and Communication Engineering from Ramaiah Institute of Technology, Bangalore. His fields of interests include artificial intelligence, big data analytics and signal processing.

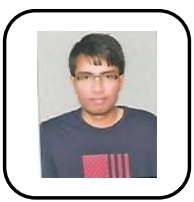

Yathin V Patil holds his BE degree in Electronics and Communication Engineering from Ramaiah Institute of Technology, Bangalore. His fields of interest include signal processing, big data analytics and mathematics 